\section{Sur les fonctions exponentielles du calcul opératoire}

par

J. G.-MIKUSIŃsKI (Wrocław).

\$ 1. Introduction. Dans le mémoire Sur les fondements du calcul opératoire ${ }^{1}$ ), j'ai exposé une méthode qui permet d'attribuer aux symboles de Heaviside un sens mathématique direct. Dans le travail présent, je vais étudier une classe de fonctions exponentielles, fournies par cette méthode, qui joue un rôle important dans les applications aux équations aux dérivées partielles.

Si $\left.k_{\epsilon} O^{2}\right)$, toute série

$$
e^{\lambda k}=1+\frac{\lambda k}{1 !}+\frac{\lambda^{2} k^{2}}{2 !}+\ldots
$$

représente une fonction exponentielle ${ }^{3}$ ). En remplaçant $k$ par 1 , la formule (1) devient

$$
e^{2}=1+\frac{\lambda}{1 !}+\frac{\lambda^{2}}{2 !}+\ldots
$$

ce qui est le cas de la fonction exponentielle classique; celle-ci existe aussi dans le calcul opératoire et garde son sens inaltéré.

Plus intéressantes sont les trois fonctions exponentielles suivantes $\left.{ }^{4}\right)$ :

$$
e^{-s \lambda}, e^{-\sqrt{s} \lambda} \text { et } e^{s\{\ln t\} \lambda}
$$

remarquons que la série analogue à (1) est, pour $e^{-s \lambda}$, divergente;

1) Studia Mathematica 11 (1949), p. 41-70.

2) Cf. loc. cit., p. 43 .

3) Cf. loc. cit., p. 64 .

4) Cf. loc. cit., p. 59 et 60. la démonstration de la convergence dans le cas des deux fonctions restantes n'est pas élémentaire ${ }^{5}$ ).

Nous étudierons ici les fonctions exponentielles de la forme

$$
e^{-s^{\alpha_{\lambda}}},
$$

où $a$ est un nombre réel donné; pour $a=0, \frac{1}{2}, 1$, on se trouve dans l'un des cas précédents.

Nous démontrerons que, $\lambda$ étant réel, la fonction $e^{-s^{a} \lambda}$ existe pour $\alpha \leqslant 1$ et n'existe pas pour $\alpha>1$. Lorsque $\lambda$ est complexe (non réel), la fonction $e^{-s^{\alpha} \lambda}$ existe pour $\alpha<1$ et n'existe pas pour $\alpha \geqslant 1$.

§ 2. Généralités. Rappelons la définition de la fonction exponentielle: Étant donné un opérateur $w$, nous désignons par $e^{w \lambda}$ ( $\lambda$ réel) la fonction opératoire qui

$1^{0}$ est régulièrement dérivable dans tout intervalle fini $\alpha \leqslant \lambda \leqslant \beta$;

$2^{0}$ satisfait à l'équation différentielle

$$
x^{\prime}(\lambda)=w x(\lambda)
$$

$3^{0}$ admet la valeur 1 pour $\lambda=0$.

La fonction exponentielle $e^{w \lambda}$, si elle existe, est univoquement définie par l'opérateur $w$. Or, elle peut ne pas exister pour certaines valeurs de $w$, comme nous allons le montrer dans la suite.

Le critère suivant nous sera utile:

Pour qu'il existe $e^{w \lambda}$, ò̀ $w=b / a \quad(a, b \in C, a \neq 0)$, il faut et il suffit qu'il existe dans un domaine

$$
\lambda_{1} \leqslant \lambda \leqslant \lambda_{2}, \quad t \geqslant 0
$$

une fonction de deux variables $x(\lambda, t)$, non identiquement nulle, possédant dans ce domaine la dérivée partielle continue $x_{2}(\lambda, t)$ et satisfaisant à l'équation intégro-différentielle

$$
\int_{0}^{t} a(t-\tau) x_{\lambda}(\lambda, \tau) d \tau=\int_{0}^{t} b(t-\tau) x(\lambda, \tau) d \tau
$$

La nécessité de la condition. L'existence de $e^{w 2}$ entraîne celle d'une fonction paramétrique non nulle $y(\lambda)$ qui possède une dérivée $y^{\prime}(\lambda)$ étant encore une fonction paramétrique, telle que

$$
a y^{\prime}(\lambda)=b y(\lambda) \text {. }
$$

5) J. G.-Mikusiński et C. Ryll-Nardzewski, Sur l'opérateur de translation, ce volume, p. 205; C. Ryll-Nardzewski, Sur les séries de puissances de l'opérateur différentiel (à parâ̂tre dans Studia Mathematica 13).

Studia Mathematica. T. XII. 
La dérivée $y^{\prime}(\lambda)$ n'est pas continue en général. Or, en posant

on a encore

$$
x(\lambda)=a y(\lambda),
$$

$$
a x^{\prime}(\lambda)=b x(\lambda)
$$

et la dérivée $x^{\prime}(\lambda)=b y(\lambda)$ est évidemment continue. La nécessité de la condition se trouve done démontrée.

La suffisance découle aussitôt d'un critère analogue, démontré dans le mémoire précité ${ }^{6}$ ).

Nous ajouterons encore quelques remarques générales.

S'il existe $e^{w \pi}$, on a $e^{w \lambda_{1}} \cdot e^{w \lambda_{2}}=e^{w\left(\lambda_{1}+\lambda_{2}\right)}$ pour tous $\lambda_{1}$ et $\lambda_{2}$ réels.

S'il existe deux fonctions $e^{w_{1} \lambda}$ et $e^{w_{2} \lambda}$, la fonction $e^{\left(\alpha_{1} w_{1}+\alpha_{2} w_{2}\right) \lambda}$ existe, quels que soient les nombres réels $\alpha_{1}$ et $\alpha_{2}$, et elle est égale au produit $e^{a_{1} w_{1} \lambda} \cdot e^{\alpha_{2} w_{2} \lambda}$.

En particulier, s'il existe $e^{w \lambda}$ et $e^{i w \lambda}$, il existe $e^{\beta w \lambda}$ pour tout $\beta$ complexe. Dans ce cas, il y a lieu de considérer la fonction $e^{w \lambda}$ dans le domaine complexe de $\lambda$.

§ 3. L'existence de $e^{-s^{\alpha} \lambda}$ pour $\alpha \leqslant 0$. Si $\alpha \leqslant-1$, on a $s^{\alpha}=l^{-\alpha} \epsilon C$ et la fonction exponentielle $e^{-s^{a} \lambda}$ peut s'écrire sous la forme de la série convergente, pour tout $\lambda$ complexe,

$$
e^{-s^{\alpha} \lambda}=1-\frac{\lambda l^{-\alpha}}{1 !}+\frac{\lambda^{2} l^{-2 \alpha}}{2 !} \mp \ldots
$$

Si $-1<\alpha<0$, la série (4) est encore convergente pour tout $\lambda$ complexe, e'est ce qui résulte du lemme général suivant:

Lemme 1. Si le rayon de convergence de la série aux coefficients numériques

$$
\Phi(\lambda)=\alpha_{0}+\alpha_{1} \lambda+\alpha_{2} \lambda^{2}+\ldots
$$

n'est pas nul et si $k=\{k(t)\}$ est une fonction continue pour $t$ positif et telle que

$$
|k(t)|<t^{-\eta}
$$

au voisinage de $t=0$, alors la série

$$
\Phi(\lambda k)=\alpha_{0}+a_{1} \lambda k+a_{2} \lambda^{2} k^{2}+\ldots
$$

5) Cf. loc. cit., p. 56. Ce critère ne diffère du précédent que par ce que la supposition de continuité de $x^{\prime}(\lambda)=\left\{x_{\lambda}(\lambda, t)\right\}$ y est supprimée. L'énoncé dn présent article est commode dans les applications. converge pour tout $\lambda$ complexe; on a en outre

$$
[\Phi(\lambda k)]^{\prime}=\alpha_{1} k+2 \alpha_{2} \lambda k^{2}+\ldots,
$$

et la dernière série converge encore pour tout $\lambda$ complexe.

Dans le cas, où $k$ est continue pour tout $t \geqslant 0$, ce lemme a été démontré dans le travail précité (page 63). Si $k$ n'est pas continue au point $t=0$, il existe un nombre naturel $n$ tel que $k^{n} \in C$. En effet, si $k^{n}=\left\{k_{n}(t)\right\}$, on a au voisinage de 0

$$
|k(t)|<\frac{\Gamma(1-\eta)^{n}}{\Gamma(n-n \eta)} t^{n-n \eta-1},
$$

où $\Gamma$ désigne la fonction gamma d'Euler; il suffit évidemment de prendre pour $n$ un entier tel que $n-n \eta-1>0$.

Écrivons

$$
\Phi(\lambda k)=\sum_{\nu=0}^{\infty} \alpha_{\nu}(\lambda k)^{\nu}=\sum_{\mu=0}^{n-1}\left[\alpha_{\mu}(\lambda k)^{\mu}+(\lambda k)^{\mu} \sum_{\nu=1}^{\infty} \alpha_{v n+\mu}\left(\lambda^{n} k^{n}\right)^{\nu}\right]
$$

chacune des séries $\sum_{y=1}^{\infty} \alpha_{\nu n+\mu}\left(\lambda^{n} k^{n}\right)^{\nu} \quad(\mu=0, \ldots, n-1)$ est convergente, car $k^{n} \epsilon C$ et chacune des séries $\sum_{\nu=1}^{\infty} \alpha_{v n+\mu} \lambda^{\nu}$ a un rayon de convergence non nul. II s'ensuit que la série $\Phi(\lambda k)$ converge. On voit aussi facilement que la formule pour $[\Phi(\lambda k)]^{\prime}$ a lieu, ce qui complète la démonstration.

Ce lemme peut aussi. être démontré directement.

Si enfin $\alpha=0$, la série (4) se réduit à la formule classique (2).

Nous avons ainsi démontré que si $\alpha \leqslant 0$, la fonction $e^{-s^{a_{\lambda}}}$ existe pour tout $\lambda$ complexe et peut être représentée par la série convergente (4).

Remarquons encore que, dans le cas $\alpha<0$, on peut écrire explicitement

$$
e^{-s^{\alpha} \alpha}=1+\left\{\sum_{\nu=1}^{\infty}(-1)^{\nu} \frac{t^{-\nu a-1}}{\nu ! \Gamma(-\nu \alpha)}\right\}
$$

§ 4. L'existence de $e^{-s^{\alpha} \lambda}$ pour $0<\alpha<1$. Nous démontrerons le théorème suivant:

Si $0<\alpha<1$, la fonction exponentielle $e^{-s^{\alpha} \lambda}$ existe pour tout $\lambda$ complexe. Pour $\lambda$ réel et positif, elle se réduit à une fonction paramétrique réelle $\{F(\lambda, t)\}$; les dérivées partielles $\partial^{n} F(\lambda, t) / \partial t^{n}(n=1,2, \ldots)$ exis- 
tent pour tout $t=0$ et elles s'annulent toutes au point $t=0$, ainsi que la fonction $F$ elle-même; dans ce cas on peut écrire explicitement

(5) $\quad F(\lambda, t)=\frac{1}{\pi} \int_{0}^{\infty} \exp \left(-t u-\lambda \cos \alpha \pi \cdot u^{\alpha}\right) \cdot \sin \left(\lambda \sin \alpha \pi \cdot u^{\alpha}\right) d u$.

La démonstration s'appuyera sur la transformation de Laplace. Fixons $\alpha$ arbitrairement dans l'intervalle $0<\alpha<1$ et considérons la fonction des trois variables réelles $\lambda>0, \mu$ et $t \geqslant 0$, définie par l'intégrale

$$
f(\lambda, \mu, t)=\frac{1}{2 \pi i} \int_{L} z^{\mu} \exp \left(z t-z^{a} \lambda\right) d z
$$

prise le long de la droite $\mathrm{R}(z)=1$. Cette intégrale converge absolument et uniformément dans tout domaine

$$
\text { (6) } \quad 0<\lambda_{0} \leqslant \lambda, \quad \mu \leqslant \mu_{0}, \quad 0 \leqslant t \leqslant t_{0} \text {; }
$$

on a en effet pour $\mathrm{R}(z)=1$

$$
\left|z^{\mu} \exp \left(z t-z^{\alpha} \lambda\right)\right| \leqslant|z|^{\mu_{0}} \exp \left[t_{0}-|z|^{\alpha} \cos \left(\alpha \pi \lambda_{0} / 2\right)\right]
$$

et l'intégrale

$$
\int_{L}|z|^{\mu_{0}} \exp \left[\mathrm{t}_{0}-|z|^{\alpha} \cos \left(\alpha \pi \lambda_{0} / 2\right)\right] d z
$$

converge. Il s'ensuit que la fonction $f(\lambda, \mu, t)$ est continue. De plus elle est réelle, car l'expression sous le signe d'intégrale admet les valeurs conjuguées pour celles de $z$.

On a les formules suivantes:

$$
\begin{gathered}
\frac{\partial^{n}}{\partial t^{n}} f(\lambda, \mu, t)=f(\lambda, \mu+n, t), \\
\int_{0}^{t} \frac{(t-\tau)^{\alpha-1}}{\Gamma(\alpha)} f(\lambda, \mu+\alpha, \tau) d \tau=f(\lambda, \mu, t), \\
\frac{\partial}{\partial \lambda} f(\lambda, \mu, t)=-f(\lambda, \mu+\alpha, t) .
\end{gathered}
$$

Les formules (7) et (9) sont évidentes; pour démontrer (8), remarquons que

$$
\int_{0}^{\infty} e^{-z t} f(\lambda, \mu+\alpha, t) d t=z^{\mu+a} \exp \left(-z^{\alpha} \lambda\right)
$$

$$
\begin{gathered}
\int_{0}^{\infty} e^{-z t} t^{\alpha-1} d t=z^{-\alpha} \Gamma(\alpha), \\
\int_{0}^{\infty} e^{-z t} f(\lambda, \mu, t) d t=z^{\mu} \exp \left(-z^{\alpha} \lambda\right) ;
\end{gathered}
$$

cela étant, la formule (8) s'obtient facilement à l'aide du théorème connu de Borel sur le produit de composition.

En posant $f(\lambda, \mu)=\{f(\lambda, \mu, t)\}$ et revenant à la notation du calcul opératoire, on tire de (8) et $(9)$

ou bien

$$
l^{\alpha} f_{2}(\lambda, \mu)=-f(\lambda, \mu)
$$

$$
f_{\lambda}(\lambda, \mu)=-s^{\alpha} f(\lambda, \mu),
$$

ce qui prouve l'existence de la fonction exponentielle $e^{-s^{\alpha} \lambda}$ pour $\lambda$ réel (et $0<\alpha<1$ ).

D'après une remarque que je dois à C. RYLL-NARDZEWSKI, cette démonstration peut être étendue aux valeurs complexes de $\lambda$ de la manière suivante. Supposons, un moment, que $\lambda$ soit réel et posons

$$
g(\lambda, \mu, t)=\frac{1}{2 \pi i} \int_{L} z^{\mu} \exp \left(z t-e^{\omega i} z^{\alpha} \lambda\right) d z
$$

où $\omega$ est un nombre positif. On a pour $\mathrm{R}(z)=1$

$$
\left|z^{\mu} \exp \left(z t-e^{\omega i} z^{\alpha} \lambda\right)\right|=|z|^{\mu_{0}} \exp \left[t_{0}-|z|^{\alpha} \lambda_{0} \cos (\alpha \pi / 2+\omega)\right]
$$

dans le domaine (6). En choisissant $\omega$ assez petit, l'intégrale (10) sera absolument et uniformément convergente dans (6). De la même façon que tout à l'heure, on démontrera l'existence de la fonction exponentielle $e^{-e^{\omega i_{s} \alpha_{\lambda}}}$. Or, l'existence des deux fonctions

$$
e^{-s^{a} \lambda} \quad \text { et } \quad e^{-e^{\omega i} s^{\alpha} \lambda}
$$

entraîne l'existence de $e^{-\zeta s^{\alpha} \lambda}$ pour tout $\zeta$ complexe. En effet, on peut écrire $\zeta=\xi+\eta e^{\omega i}$, où $\xi$ et $\eta$ sont réels, et

$$
e^{-\zeta s^{\alpha} \lambda}=e^{-s^{\alpha} \xi \lambda} \cdot e^{-e^{\omega i s_{s}^{\alpha} \eta \lambda} .}
$$

L'existence de $e^{-s^{\alpha} \lambda}(0<\alpha<1)$ se trouve donc démontrée pour tout $\lambda$ complexe. 
Remarquons maintenant que pour $\lambda$ réel et positif la fonction $e^{-s^{a} \lambda}(0<\alpha<1)$ se réduit à une fonction paramétrique réelle $\left.{ }^{7}\right)$. En effet, en vertu de (9), on a dans ce cas

$$
e^{-s^{\alpha} \lambda}=\{f(\lambda, 0, t)\}=\left\{\frac{1}{2 \pi i} \int_{L} \exp \left(z t-z^{\alpha} \lambda\right) d z\right\} .
$$

Cette fonction est réelle, car l'expression sous le signe d'intégrale admet des valeurs conjuguées pour les valeurs conjuguées de $z$. On peut facilement obtenir pour $F(\lambda, t)=f(\lambda, 0, t)$ une formule réelle. Remarquons d'abord que la ligne d'intégration peut être remplacée dans l'intégrale (10) par l'axe imaginaire. En effet, l'intégrale

$$
\int \exp \left(z t-z^{\alpha} \lambda\right) d z
$$

prise le long du contour $A B C D E F A$ est nulle. Si le rayon du demi-cercle $D E$ décroit indéfiniment, l'intégrale correspondante s'annule. Pareillement, les intégrales le long des segments $B C$ et $F A$ tendent vers zéro, lorsque ces segments s'éloignent indéfiniment. On a donc

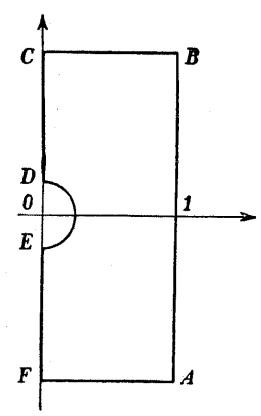

$$
F(\lambda, t)=\frac{1}{2 \pi i} \int_{-i \infty}^{+i \infty} \exp \left(z t-z^{a} \lambda\right) d z
$$

$(\lambda>0, t \geqslant 0)$

En séparant les parties réelle et imaginaire sous le signe d'intégrale, on en tire sans peine la formule (5) ${ }^{8}$ ).

Il reste à démontrer que la fonction $F$ ainsi que toutes ses dérivées $\partial^{n} F / \partial t^{n} \quad(n=1,2, \ldots)$ sont nulles pour $t=0$. En vertu de (7), il suffit évidemment de montrer que

$$
f(\lambda, \mu, 0)=0
$$

${ }^{7)}$ Cf. loc. cit., p. 51 .

8) La ligne d'intégration dans la formule (11) peut encore être modifiée de diverses façons; on peut ainsi parvenir à diverses formules pour $F(\lambda, t)$. Nous nous bornerons à en reproduire (sans démonstration) la suivante:

$$
F(\lambda, t)=\frac{1}{\pi} \int_{0}^{\infty} \exp \left(-u t-u^{\alpha} \lambda \cos \alpha \pi\right) \cdot \sin \left(u^{\alpha} \lambda \sin \alpha \pi\right) d u .
$$

pour tout $\lambda>0$ et $\mu$ réel. En effet, l'intégrale

$$
\int_{A B C A} z^{\mu} \exp \left(-z^{\alpha} \lambda\right) d z,
$$

prise le long du contour formé du segment $A B$ de la droite $L$ et de l'arc $B C A \mathrm{du}$ cercle de centre $z=0$, est nulle et on peut écrire

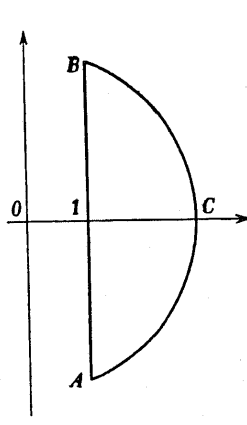
$\int_{A B}=\int_{A B C}$. Lorsque le rayon du cercle croît indéfiniment, l'intégrale $\int_{A C B}$ tend vers zéro et, par conséquent, il en est de même de l'intégrale $\int$, ce qui prouve l'égalité (12).

§ 5. La non-existence de $e^{-s^{a_{2}}}$ pour $\alpha>1$. Nous démontrerons d'abord que $l a$ fonction $e^{-s^{a_{\lambda}}}$ n'existe pas, lorsque $\alpha>1$ et $\lambda$ est réel. Dans ce but il suffit de montrer que, pour l'équation intégro-différentielle (entendue au sens ordinaire)

$$
\int_{0}^{t} \frac{(t-\tau)^{\alpha-1}}{\Gamma(\alpha)} x_{\lambda}(\lambda, \tau) d \tau+x(\lambda, t)=0
$$

considérée dans un rectangle $R:\left(\lambda_{1} \leqslant \lambda \leqslant \lambda_{2}, 0 \leqslant t \leqslant t_{0}\right)$, toute solution continue $x(\lambda, t)$ possédant la dérivée partielle $x_{\lambda}(\lambda, \tau)$ continue, est identiquement nulle.

Nous nous appuyerons sur le lemme suivant:

Lemme 2. Si les fonctions $f(t)$ et $g(t)$ (réelles ou complexes) sont sommables dans l'intervalle $(0, T)$ et si $\left.h(t)=\int_{0}^{t} f(t-\tau) g(\tau) d \tau^{9}\right)$, alors on $a$

$$
\int_{0}^{T} e^{-\sigma t} h(t) d t=\int_{0}^{T} e^{-\sigma t} f(t) d t \cdot \int_{0}^{T} e^{-\sigma t} g(t) d t-e^{-\sigma T} \cdot M(\sigma),
$$

où

$$
M(\sigma)=\iint_{D} e^{-\sigma(u+v-T)} f(u) g(v) d u d v,
$$

le domaine d'intégration $D$ étant défini par les inégalités

$$
0 \leqslant T-u \leqslant v \leqslant T .
$$

9) La sommabilité de $f$ et $g$ entraîne celle de $h$; voir J. G.-Mikusiński, L'anneau algébrique et ses applications dans l'analyse fonctionnelle, Aunales Universitatis Mariae Curie-Skłodowska, Lublin, A I (1947), p. 9. 
Ce lemme est une modification du théorème connu de Borel et se démontre facilement par la substitution $u=t-\tau, v=\tau$.

Supposons maintenant que $x(\lambda, t)$ soit une solution de (12) pourvue de dérivée partielle $x_{\lambda}(\lambda, t)$ continue. Posons

$$
X(\lambda, \sigma)=\int_{0}^{T(\lambda)} e^{\sigma[T(\lambda)-t]} x(\lambda, t) d t,
$$

où $T(\lambda)$ est une fonction continûment dérivable et non-négative dans l'intervalle $\left[\lambda_{1}, \lambda_{2}\right]$ tel que $T\left(\lambda_{1}\right)=T\left(\lambda_{2}\right)=0$.

D'après le lemme 2 , on a alors

$$
A(\lambda, \sigma) \cdot \int_{0}^{T(\lambda)} e^{\sigma[T(\lambda)-t]} x_{\lambda}(\lambda, t) d t+X(\lambda, \sigma)=M(\lambda, \sigma),
$$

où

$$
A(\lambda, \sigma)=\int_{0}^{T(\lambda)} e^{-\sigma t} \frac{t^{a-1}}{\Gamma(\alpha)} d t
$$

et

$$
M(\lambda, \sigma)=\iint_{D(\lambda)} e^{-\sigma[u+v-T(\lambda)]} \frac{n^{\alpha-1}}{\Gamma(\alpha)} x_{\lambda}(\lambda, v) d u d v ;
$$

le domaine d'intégration est ici défini par les inégalités

$$
0 \leqslant T(\lambda)-u \leqslant v \leqslant T(\lambda) .
$$

On voit facilement que la fonction $M(\lambda, \sigma)$ est bornée dans le domaine $\sigma \geqslant 0, \lambda_{1} \leqslant \lambda \leqslant \lambda_{2}$, car

$$
|M(\lambda, \sigma)| \leqslant \iint_{D} \frac{u^{a-1}}{\Gamma(\alpha)}\left|x_{\lambda}(\lambda, v)\right| d u d v
$$

où $D$ est le carré $0 \leqslant u, v \leqslant \operatorname{Max}_{\lambda_{1} \leqslant \lambda \leqslant \lambda_{0}}|T(\lambda)|$.

Il est aisé de vérifier que

$$
X_{\lambda}(\lambda, \sigma)=T^{\prime}(\lambda) \cdot x(\lambda, T(\lambda))+\sigma T^{\prime}(\lambda) X(\lambda, \sigma)+\int_{0}^{T(\lambda)} e^{\sigma[T(\lambda)-t]} x_{\lambda}(\lambda, t) d t .
$$

On a donc, en vertu de (13),

$$
\begin{gathered}
A(\lambda, \sigma) \cdot X_{\lambda}(\lambda, \sigma)+\left[1-\sigma T^{\prime}(\lambda) A(\lambda, \sigma)\right] X(\lambda, \sigma) \\
=M(\lambda, \sigma)-T^{\prime}(\lambda) x(\lambda, T(\lambda)) .
\end{gathered}
$$

En vertu de l'inégalité

$$
0 \leqslant A(\lambda, \sigma) \leqslant \int_{0}^{\infty} e^{-\sigma t} \frac{t^{a-1}}{\Gamma(\alpha)} d t=\frac{1}{\sigma^{\alpha}}
$$

il existe done un nombre $S$ tel que

$$
1-\sigma T^{\prime}(\lambda) A(\lambda, \sigma)>\frac{1}{2}
$$

pour $\sigma>S$.

Fixons arbitrairement $\sigma>S$ et désignons par $\lambda_{\sigma}$ le point de l'intervalle $\left[\lambda_{1}, \lambda_{2}\right]$, où le module de $X(\lambda, \sigma)$ atteint son maximum.

On a alors $X_{\lambda}\left(\lambda_{\sigma}, \sigma\right)=0, \quad$ car $X\left(\lambda_{1}, \sigma\right)=X\left(\lambda_{2}, \sigma\right)=0$. D'après (14) et (15), on a donc

$$
|X(\lambda, \sigma)| \leqslant\left|X\left(\lambda_{\sigma}, \sigma\right)\right| \leqslant 2\left|M\left(\lambda_{\sigma}, \sigma\right)-T^{\prime}\left(\lambda_{\sigma}\right) x\left(\lambda_{\sigma}, T\left(\lambda_{\sigma}\right)\right)\right| \leqslant N,
$$

où le nombre $N$ ne dépend que du choix de la fonction $T(\lambda)$.

En écrivant cette inégalité explicitement,

$$
\left|\int_{0}^{T(\lambda)} e^{\sigma[T(\lambda)-t]} x(\lambda, t) d t\right| \leqslant N
$$

on conclut d'après un théorème des moments $\left.{ }^{10}\right)$ que $x(\lambda, t)=0$ identiquement dans le domaine

$$
\lambda_{2} \leqslant \lambda \leqslant \lambda_{2}, \quad 0 \leqslant t \leqslant T(\lambda)
$$

Or, la fonction $T(\lambda)$ peut être choisie arbitrairement, done $x(\lambda, t)=0$ identiquement pour $\lambda_{1} \leqslant \lambda \leqslant \lambda_{2}, t \geqslant 0$.

La démonstration de la non-existence de $e^{-8^{a} \lambda}$ pour $\alpha>1$ et $\lambda$ réel est donc achevée.

$\Pi$ est maintenant facile d'étendre la démonstration pour tout $\lambda$ complexe mais non imaginaire, e'est-à-dire pour tout $\lambda$ de la forme $\lambda_{1}+i \lambda_{2}$, où $\lambda_{1}$ et $\lambda_{2}$ sont réels et $\lambda_{1} \neq 0$.

10) Voir J. G.-Mikusiński, Remarks on the moment problem and a theorem of Picone, Colloquium Mathematicum II. 2, p. 138-14I et J. G.-Mikusiński, A theorem on moments, ce volume, p. 191-193. Remarquons que les inégalités

$$
\int_{0}^{T} e^{\sigma(T-i)} g(t) d t \leqslant N \text { et } \int_{1}^{b} x^{\sigma} f(x) d x \leqslant N
$$

sont équivalentes par la substitution $e^{T-t}=x, b=e^{T}, g(t)=f(x)$. 
Supposons par contre qu'existe la fonction $x(\lambda)=e^{\beta_{s} a_{2}}$ pour un certain $\beta=\beta_{1}+i \beta_{2}$, où $\beta_{1} \neq 0(\alpha>1, \lambda$ réel). Cette fonction satisfait à l'équation différentielle

$$
x^{\prime}(\lambda)=\beta s^{\alpha} x(\lambda)
$$

avec la condition $x(0)=1$. Or, la fonction conjuguée $\left.\bar{x}(\lambda)^{11}\right)$ satisfait évidemment à l'équation

$$
\bar{x}^{\prime}(\lambda)=\bar{\beta} s^{a} \bar{x}(\lambda),
$$

et l'on a encore $x(0)=1 ; x(\lambda)$ est done la fonction exponentielle $e^{\bar{\beta}^{a} \lambda}$ et, par conséquent, il existe

$$
e^{(\beta+\bar{\beta})^{\alpha} \lambda}=e^{\beta s^{\alpha} \lambda} \cdot e^{\bar{\beta}^{\alpha} \lambda},
$$

ce qui est en contradiction avec ce que nous venons de démontrer, car $\beta+\bar{\beta}$ est réel.

Le théorème de non-existence de $e^{s^{\alpha_{\lambda}}}(\alpha>1)$ est encore valable pour les valeurs imaginaires de $\lambda$, mais la démonstration exige une autre méthode. Nous la présenterons au $\S 7$; elle s'appuyera sur un théorème des moments que nous allons énoncer maintenant.

8 6. Un théorème des moments et son application au caleul opératoire. Voici le théorème:

(I) Soit $f(x)$ une fonction (au sens ordinaire) sommable sur l'intervalle donné $0<a<x<b$. Soit $x$ un nombre positif $\leqslant 1$ et $\mu$ un nombre positif quelconque. S'il existe, pour tout $c>a$, un nombre $M$ tel que

$$
\left|\int_{a}^{b} x^{\mu n^{x}} f(x) d x\right|<M c^{\mu n^{x}} \quad(n=1,2, \ldots)
$$

on a $f(x)=0$ presque partout dans $(a, b)$.

11) Tout opérateur $a$ se laisse représenter sous la forme $a=(p+i q) / r$ où $p, q$ et $r$ sont des fonctions réelles de $C$. On pose par définition $\bar{a}=(p-i q) / r$ les opérateurs $a$ et $\vec{a}$ sont dits conjugués. Si, en particulier, $q=0$, l'opérateur est réel. L'opérateur $s^{\alpha}$ est réel, car il peut être représenté sous la forme

$$
\delta^{a}=\frac{\{\Gamma(a+1)\}}{\left\{t^{a}\right\}} .
$$

Ce théorème, démontré dans l'un de mes articles antérieurs ${ }^{12}$ ), entraîne le théorème suivant:

(II) Soit $g$ une fonction de $C$. Soient $x$ un nombre positif $\leqslant 1$ et $\mu$ un nombre positif quelconque. S'il existe pour tout $\varepsilon>0$ une fonction h. $\mathrm{CO}$ telle que

$$
\left|\frac{g}{s-\mu n^{\varkappa}}\right| \leqslant h \cdot \exp \left(\varepsilon n^{*}\right) \quad(n=1,2, \ldots)
$$

on $a g=0$.

Remarque. Les notions de module et d'inégatité n'ont pas été introduites, jusqu'à présent, pour les opérateurs. Or, les opérateurs qui paraissent dans l'inégalité (16) ne sont que des fonctions et les deux symboles (de module et d'inégalité) sont à entendre au sens ordinaire.

Si $f g$ est le produit de composition de $f$ et $g$, il est aisé de voir que l'on a généralement $|f g| \leqslant|f| \cdot|g|$, où $|f| \cdot|g|$ est le produit de composition de $|f|$ et $|g|$.

Ecrivons l'inégalité (16) sous la forme

$$
\left|\int_{0}^{t} e^{\mu n^{*} \tau} g(t-\tau) d \tau\right| \leqslant h(t) e^{\varepsilon n^{\varkappa}} \quad(t \geqslant 0 ; n=1,2, \ldots) .
$$

Fixons $t>0$ arbitrairement et posons

$$
x=e^{\tau}, \quad f(x)=g(t-\tau), \quad a=1, \quad b=e^{t}
$$

et

$$
c^{\mu}=e^{\varepsilon}, \quad M=h(t) ;
$$

alors l'inégalité (17) se transforme en l'inégalité (15), ce qui prouve le théorème (II).

Le corollaire suivant sera utile au $\S 7$ :

Corollaire. Soit $g$ une fonction de $C$. Si

$$
\left|\frac{g}{s^{\alpha}-\mu n}\right| \leqslant n^{\nu} h \quad(n=1,2, \ldots),
$$

ò̀ $\alpha \geqslant 1, \mu>0, v \geqslant 0$ et $h \epsilon C$, on a $g=0$.

12) J. G.-Mikusiński, A theorem on moments, ce volume, p. 191-193. 
On a en effet

$$
\text { (20) } \quad\left|\frac{l^{a} g}{s-\sqrt[a]{\mu n}}\right| \leqslant \frac{1-\mu n l^{a}}{s-\sqrt[a]{\mu n}} \cdot\left|\frac{g}{s^{a}-\mu n}\right| \text {; }
$$

il est facile de vérifier que

$$
\frac{1-\mu n l^{\alpha}}{s-\sqrt[\alpha]{\mu n}}=\left(1-\mu n l^{\alpha}\right)\{\exp \sqrt[\alpha]{\mu n} t\}=\left\{\frac{\mu n}{\Gamma(\alpha)} \int_{i}^{\infty} \exp [-\sqrt[\alpha]{\mu n}(\tau-t)] \cdot \tau^{\alpha-1} d \tau\right\}
$$

d'où

$$
\begin{gathered}
0 \leqslant \frac{1-\mu n l^{\alpha}}{s-\sqrt[a]{\mu n}} \leqslant\left\{\frac{\mu n}{\Gamma(\alpha)} \int_{t}^{\infty} \exp [-\sqrt[a]{\mu}(\tau-t)] \cdot \tau^{\alpha-1} d \tau\right\} \\
=\left\{\frac{\mu n}{\Gamma(\alpha)} \exp (\sqrt[a]{\mu} t) \int_{t}^{\infty} \exp (-\sqrt[\alpha]{\mu} \tau) \cdot \tau^{\alpha-1} d \tau\right\} \leqslant \frac{n}{s-\sqrt[\alpha]{\mu}} .
\end{gathered}
$$

Si $a, \mu$ et $\nu$ sont donnés, il existe, pour tout $\varepsilon>0$, un nombre $\gamma$ tel que

$$
n^{\nu+i} \leqslant \gamma \cdot \exp (\varepsilon \sqrt[a]{\mu n}) \quad(n=1,2, \ldots)
$$

D'après les inégalités (19), $(20),(21)$, et $(22)$, il vient

$$
\left|\frac{l^{\alpha} g}{s-\sqrt[a]{\mu n}}\right| \leqslant \frac{\gamma h}{s-\sqrt[a]{\mu}} \exp (\varepsilon \sqrt[a]{\mu n}) \quad(n=1,2, \ldots)
$$

En vertu du théorème (II), on a donc $l^{\alpha} g=0$, et, par conséquent, $g=0$, ce qu'il fallait démontrer.

\$ 7. La non-existence de $e^{-8^{\alpha} \lambda}$ pour $\alpha \geqslant 1$ et $\lambda$ imaginaire. $\Pi$ suffit de démontrer que toute fonction paramétrique satisfaisant à l'équation

$$
x^{\prime}(\lambda)=-i s^{\alpha} x(\lambda) \quad(\alpha \geqslant 1)
$$

où $\lambda$ est réel, est nulle.
Soient $\lambda_{1}$ et $\lambda_{2}\left(\lambda_{1}<\lambda_{2}\right)$ deux nombres réels quelconques. Multiplions l'égalité (23) par $e^{i \omega_{n}^{*} \lambda}$, où $\omega_{n}=2 n \pi /\left(\lambda_{2}-\lambda_{1}\right)$, et intégrons la entre les limites $\lambda_{1}$ et $\lambda_{2}$ :

$$
\int_{\lambda_{1}}^{\lambda_{2}} \exp \left(i \omega_{n} \lambda\right) x^{\prime}(\lambda) d \lambda=-i s^{\alpha} \int_{\lambda_{1}}^{\lambda_{2}} \exp \left(i \omega_{n} \lambda\right) x(\lambda) d \lambda
$$

En effectuant l'intégration per partes, on a

$$
\begin{gathered}
\int_{\lambda_{1}}^{\lambda_{2}} \exp \left(i \omega_{n} \lambda\right) x^{\prime}(\lambda) d \lambda \\
=\exp \left(i \omega_{n} \lambda_{2}\right) x\left(\lambda_{2}\right)-\exp \left(i \omega_{n} \lambda_{1}\right) x\left(\lambda_{1}\right)-i \omega_{n} \int_{\lambda_{1}}^{\lambda_{2}} \exp \left(i \omega_{n} \lambda\right) x(\lambda) d \lambda .
\end{gathered}
$$

Or, $\exp \left(i \omega_{n} \lambda_{1}\right)=\exp \left(i \omega_{n} \lambda_{2}\right)$ pour $n=1,2, \ldots$, donc

$$
\exp \left(i \omega_{n} \lambda_{2}\right)\left[x\left(\lambda_{2}\right)-x\left(\lambda_{1}\right)\right]=-i\left(s^{\alpha}-\omega_{n}\right) \int_{\lambda_{2}}^{\lambda_{1}} \exp \left(i \omega_{n} \lambda\right) x(-\lambda) d \lambda
$$

et

$$
\frac{x\left(\lambda_{2}\right)-x\left(\lambda_{1}\right)}{s^{\alpha}-\omega_{n}}=-i e^{-i \omega_{n} \lambda_{2}} \int_{\lambda_{1}}^{\lambda_{2}} \exp \left(i \omega_{n} \lambda\right) x(\lambda) d \lambda,
$$

d'où

$$
\left|\frac{x\left(\lambda_{2}\right)-x\left(\lambda_{1}\right)}{s-\frac{2 n \pi}{\lambda_{2}-\lambda_{1}}}\right| \leqslant \int_{\lambda_{1}}^{\lambda_{2}}|x(\lambda)| d \lambda .
$$

En vertu du Corollaire du $\S 6$, on a donc

$$
x\left(\lambda_{2}\right)-x\left(\lambda_{1}\right)=0
$$

comme $\lambda_{1}$ et $\lambda_{2}$ sont arbitraires, il s'ensuit que la fonction $x(\lambda)$ est constante, donc, en vertu de (23), identiquement nulle.

$\$ 8$. Le cas limite $\alpha=1$. Si $\lambda$ est réel, la fonction $e^{-8 \lambda}$ représente l'opérateur de translation bien connu. Si $\lambda$ est imaginaire, cette fonction n'existe pas, d'après le paragraphe précédent. Pour compléter la discussion il reste à considérer le cas: $\lambda=\lambda_{1}+i \lambda_{2}$, où $\lambda_{1}$ et $\lambda_{2}$ sont réels et non nuls. Or, dans ce cas la fonction ex- 
ponentielle n'existe pas. En effet, si elle existait, alors existerait aussi la fonction

ce qui n'a pas lieu.

$$
e^{-i s \lambda_{2}}=e^{s \lambda_{1}} / e^{s\left(\lambda_{1}+i \lambda_{2}\right)},
$$

§ 9. Tableau d'existence. Les résultats principaux sur l'existence de $e^{-s^{a} \lambda}$ peuvent être illustrés par le tableau ci-dessous:

\begin{tabular}{|c|c|c|}
\hline$e^{-s^{a_{\lambda}}}$ & $\lambda$ réel & $\lambda$ non rée \\
\hline$a<1$ & \multicolumn{2}{|c|}{ Domaine d'existence } \\
\hline$a=1$ & & \\
\hline$a>1$ & \multicolumn{2}{|c|}{ Domaine de non-existence } \\
\hline
\end{tabular}

§ 10. Fonctions exponentielles composées. Nous démontrerons encore le théorème suivant:

Soient $\alpha_{1}, \ldots, \alpha_{n} n$ nombres réels distincts et $\beta_{1}, \ldots, \beta_{n} n$ nombres complexes non nuls. Pour que la fonction exponentielle

$$
\exp \left[\left(\beta_{1} s^{\alpha_{1}}+\ldots+\beta_{n} s^{a_{n}}\right) \lambda\right]
$$

existe (pour $\lambda$ réel), il faut et il suffit qu' existe (pour $\lambda$ réel) chacune des fonctions exponentielles

$$
\exp \left(\beta_{1} s^{\alpha_{1}} \lambda\right), \ldots, \exp \left(\beta_{n} s^{\alpha_{n}} \lambda\right) .
$$

Démonstration. La suffisance de cette condition est évidente. Il reste donc à démontrer sa nécessité. Soit a un nombre positif supérieur à $\alpha_{1}, \ldots, \alpha_{n}$. Si (24) existe, alors existe dans un domaine

$$
\lambda_{1} \leqslant \lambda \leqslant \lambda_{n}, \quad t \geqslant 0,
$$

une fonction de deux variables $x(\lambda, t)$, non identiquement nulle, possédant dans ce domaine la dérivée partielle $x_{\lambda}(\lambda, t)$ continue et satisfaisant à l'équation

$$
\frac{1}{\Gamma(\alpha)} \int_{0}^{t}(t-\tau)^{\alpha-1} x_{\lambda}(\lambda, \tau) d \tau=\sum_{\nu=1}^{n} \beta_{\nu} \int_{0}^{t} \frac{(t-\tau)^{\alpha-a_{\nu}-1}}{\Gamma\left(\alpha-\alpha_{\nu}\right)} x(\lambda, \tau) d \tau .
$$

Soit $\delta$ un nombre positif, fixé arbitrairement. En posant

$$
x(\lambda, t / \delta)=y(\lambda, t),
$$

on aura

$$
\frac{1}{\Gamma(\alpha)} \int_{0}^{t}(t-\tau)^{\alpha-1} y_{\lambda}(\lambda, \tau) d \tau=\sum_{\nu=1}^{n} \beta_{\nu} \delta^{a_{\nu}} \int_{0}^{t} \frac{(t-\tau)^{\alpha-\alpha_{\nu}-1}}{\Gamma\left(\alpha-\alpha_{\nu}\right)} y(\lambda, \tau) d \tau
$$

dans le domaine (25), ce qui entraîne l'existence de la fonction exponentielle

$$
\exp \left[\sum_{\nu=1}^{n} \gamma_{\nu}\left(\beta_{1} \delta_{\nu}{ }^{a_{1}} s^{a_{1}}+\ldots+\beta_{n} \delta_{\nu}{ }^{a_{n}} s^{a_{n}}\right) \lambda\right]
$$

quels que soient les nombres réels $\gamma_{1}, \ldots, \gamma_{n}$.

On peut choisir les valeurs de $\delta_{1}, \ldots, \delta_{n}$ de manière que le déterminant

$$
\left|\begin{array}{ccc}
\delta_{1}^{a_{1}} & \ldots & \delta_{n}^{a_{1}} \\
\cdots & \ldots & \delta_{1}^{a_{n}} \\
\delta_{1}^{a_{n}} & \ldots & \delta_{n}^{a_{n}}
\end{array}\right|
$$

soit différent de zéro. Il est alors possible de déterminer les valeurs de $\gamma_{1}, \ldots, \gamma_{n}$ de manière que

$$
\sum_{\nu=1}^{n} \gamma_{\nu} \delta_{\nu}^{a_{1}}=1 \text { et } \sum_{\nu=1}^{n} \gamma_{\nu} \delta_{\nu}^{a_{\varkappa}=0} \text { pour } x=2, \ldots, n
$$

Cela étant, la fonction (26) se réduit à $\exp \left(\beta_{1} s^{\alpha_{1}^{\lambda}}\right)$, d'où, par raison de symétrie, le théorème.

§ 11. Notice historique. La discussion complète de la fonction $e^{-s^{\alpha} \lambda}$ a réussi grâce à ma collaboration avec l'école jtalienne de Mauro Picone qui s'occupe des équations aux dérivées partielles. A savoir, la démonstration de la non-existence de $e^{-s a_{\lambda}}$ se réduit, dans certạins cas particuliers, aux problèmes d'unicité de ces équations.

Si $\lambda$ est réel et $\alpha=2,3, \ldots$, la non-existence de $e^{-s^{\alpha_{\lambda}}}$ résulte d'un théorème d'unicité pour l'équation

$$
\frac{\partial x}{\partial \lambda}+\frac{\partial^{\alpha} x}{\partial t^{\alpha}}=0,
$$

qui peut être déduit d'une méthode de Mauro Picone, présentée 
dans son mémoire Nouvelles méthodes de recherche pour la détermination des intégrales des équations linéaires aux dérivées partielles $\left.{ }^{13}\right)$. Notre démonstration du $\S 5$ n'est qu'une modification de cette méthode.

La non-existence de $e^{-i s^{2} \lambda}$ pour $\lambda$ réel équivaut au théorème d'unicité suivant:

Si la fonction $x(\lambda, t)$ satisfait dans le domaine $D(0 \leqslant \lambda \leqslant 1, t \geqslant 0)$ à l'équation

$$
\frac{\partial^{2} x}{\partial \lambda^{2}}+\frac{\partial^{4} x}{\partial t^{4}}=0
$$

avec les conditions initiales $\left(\frac{\partial^{j} x}{\partial t^{j}}\right)_{t=0}=0 \quad(j=0,1,2,3)$, on a $x(\lambda, t)=0$ identiquement dans $D$.

J'ai posé ce théorème comme hypothèse dans une lettre à M. Picone; la réponse positive a été donnée par GaETANo FiCHERA $\left.{ }^{14}\right)$. Notre démonstration du $\S 7$ n'est au fond qu'une modification et extension de la méthode de G. Fichera. Cette extension a réussi grâce à l'introduction du théorème des moments (au §6) qui joue dans la démonstration un rôle capital.

19) Annales de la Société Polonaise de Mathématique 19 (1946), p. 36-61.

14) G. Fichera a renforcé le théorème en remplaçant le domaine $D$ par le domaine: $0 \leqslant \lambda \leqslant 1,0 \leqslant t<T$, où $T$ peut être fini ou infini. Son travail n'a pas encore été publié.

PANSTWOWY INSTYTUT MATHMATYCZNY INSTITUT MATHEMATIQUE DE L'ÉTAT

(Reçu par la Rédaction le 15. 1. 1951).

\section{Certains théorèmes des moments}

par

C. RYLL-NARDZEWSKI (Wroclaw).

1. On connaît le théorème suivant:

Soit la fonction $f(x)$ sommable dans l'intervalle $[0, b]$ et soit $0 \leqslant a \leqslant b$. Si

$$
\int_{0}^{b} x^{n} f(x) d x=O\left(a^{n}\right)
$$

on $a f(x)=0$ presque partout dans $\left.[a, b]^{\mathbf{1}}\right)$.

Nous présenterons ici une généralisation de ce théorème.

2. Soit $\Gamma$ un arc simple rectifiable situé dans le plan complexe et $\varrho$ un nombre tel que $\Gamma$ a au plus un point commun avec le cercle $|z| \leqslant \varrho$. Soit de plus $f(z)$ une fonction définie et sommable sur l'arc $\Gamma$. Cela posé on a le théorème suivant:

$$
\int_{\Gamma} \zeta^{n} f(\zeta) d \zeta=O\left(\varrho^{n}\right)
$$

on a $f(z)=0$ presque partout sur $\Gamma$.

Démonstration. Posons

$$
F(z)=\int_{\Gamma} \frac{f(\zeta)}{\zeta-z} d \zeta .
$$

La fonction $F(z)$ est holomorphe en déhors de $\Gamma$ et pour $z$ assez grand se développe en série de Laurent

$$
F(z)=-\sum_{n=0}^{\infty} \frac{1}{z^{n+1}} \int_{\Gamma} \zeta^{n} f(\zeta) d \zeta .
$$

1) Voir J. G.-Mikusiński, Remarks on the moment problem and a theorem of Picone, Colloquium Mathematicum II. 2, p. 138-141.

Studia Mathematica. T. XII. 